\title{
Observation of Electroweak Single Top-Quark Production with the CDF Experiment
}

\author{
Jan Lueck* (on behalf of the CDF Collaboration) \\ Karlsruhe Institute of Technology \\ E-mail: lueckefnal.gov
}

\begin{abstract}
We present the observation of electroweak single top-quark production using $3.2 \mathrm{fb}^{-1}$ of data collected by the CDF experiment. Candidate events are selected for further classification by four parallel analysis techniques: one likelihood discriminant, one matrix-element discriminant, one decision-tree discriminant, and one neural-network discriminant. These outputs are combined with a super discriminant based on a neural-network analysis in order to improve the expected sensitivity. In conjunction with one neural-network discriminant using a complementary dataset we observe a signal consistent with the standard model but inconsistent with the background-only model by 5.0 standard deviations, with a median expected sensitivity in excess of 5.9 standard deviations. We also present the most current value of the CKM matrix-element $\mathrm{V}_{t b}$.
\end{abstract}

XXth Hadron Collider Physics Symposium

November 16 - 20, 2009

Evian, France

*Speaker. 
The reasons for studying electroweak single top-quark production are compelling: the production cross section is directly proportional to the square of the CKM matrix element $\left|V_{t b}\right|$, and thus a measurement of the rate constrains fourth-generation models and other new phenomena. In the $\mathrm{SM}$, top-quarks are expected to be produced singly through $s$ - or $t$-channel exchange of a virtual $W$ boson with an expected combined production cross section of $\sigma_{s+t} \sim 2.9 \mathrm{pb}[1,2]$. Both the CDF and D0 collaborations at the Tevatron have reported observation of single top-quark production [3, 4], this document describes the latest analyses done using $3.2 \mathrm{fb}^{-1}$ of data collected with the CDF II detector leading to the observation of single top-quark production.

The event selection is based on selecting $\ell+E_{T}+$ jets events, where $\ell$ is an explicitly reconstructed electron or muon with $p_{T}>20 \mathrm{GeV}$ from the $W$ boson decay. The presence of high missing transverse energy, $E_{T}>25 \mathrm{GeV}$, and two or three energetic jets, $E_{T}>20 \mathrm{GeV}$, are also required. At least one of the jets has to be identified as a jet coming from a $b$-quark. The background has contributions from events in which a $W$ boson is produced in association with one or more heavy flavor jets, events with mistakenly $b$-tagged light-flavor jets, QCD multijet events, $t \bar{t}$ production, diboson processes, and $Z+$ jet events, as shown in Fig. 1(a). From this figure, it is clear that the signal is hidden under huge and uncertain backgrounds which make counting experiments impossible.

To overcome these challenges, a variety of multivariate techniques for separating single top-quark events from the backgrounds have been developed as described in the following. One approach [5] employs neural networks (NN) [6] which combine 11 to 18 variables into one more powerful discriminant. Among the most important ones is the output of a jet-flavor separator dedicated neural network [7]. The matrix element (ME) method [8] relies on the evaluation of event probability densities for signal and background processes based on calculations of the differential cross sections. A projective likelihood function (LF) technique [9] is used to combine information from 7 to 10 input variables to optimize the separation of the single top-quark signal from the backgrounds. A separate analysis dedicated to the search for $s$-channel single top-quark production is additional performed using this technique [10]. The boosted decision tree (BDT) analysis uses binary cuts iteratively on over 20 input variables to classify events. The MET+Jets (MJ) neural network analysis is a new analysis in CDF, using data corresponding to $2.1 \mathrm{fb}^{-1}$ of integrated luminosity, designed to select events with $Z_{T}$ and jets, while vetoing events selected by the $\ell+Z_{T}+$ jet analyses. Through its orthogonal event selection, it increases the overall CDF signal acceptance by $\sim 30 \%$.

CDF combines the NN, ME, LF, and BDT channels using a super-discriminant (SD) technique. The SD method uses a neural network trained with neuro-evolution [11] to separate the signal from the background taking as inputs the discriminant outputs of the four analyses for each event. A simultaneous fit over the two exclusive channels, MJ and SD, is performed to obtain the final combined results. For illustrative purposes, Fig. 1(b) shows the distributions of the $\ell+E_{T}+$ jets super discriminant combination. The cross section is measured using a Bayesian binned likelihood technique [12] assuming a flat non-negative prior in the cross section and integrating over the systematic uncertainties, including jet energy scale, $b$-tagging efficiencies, background modeling, lepton identification and trigger efficiencies, the amount of initial and final state radiation, PDFs, and factorization and renormalization scales. The significance is calculated as a $p$-value [12], which is the probability, assuming the absence of single top-quark production, that the value of the test statistic, $-2 \ln Q$, is more signal like than that observed in data. The $p$-value is then converted into standard deviations using the integral of one side of a Gaussian. 
The excess of signal-like events over the expected background is interpreted as observation of single top-quark production with a $p$-value of $3.10 \times 10^{-7}$, corresponding to a significance of 5.0 standard deviations. The sensitivity is defined to be the median expected significance and is found to be in excess of 5.9 standard deviations. CDF finds, assuming a top-quark mass of $175 \mathrm{GeV} / \mathrm{c}^{2}$, a value of the combined $s$ - and $t$-channel cross section of $2.3_{-0.5}^{+0.6} \mathrm{pb}$, and consequential $\left|V_{t b}\right|=$ $0.91 \pm 0.11$ (stat + syst) \pm 0.07 (theory [1]). More details can be found on the CDF public web page [13].

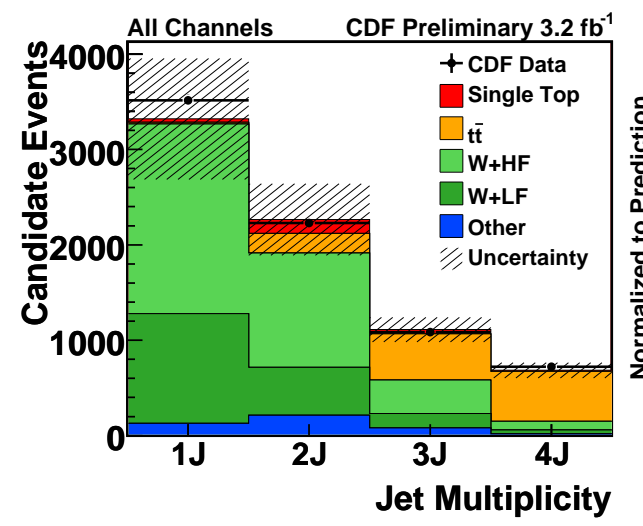

(a)

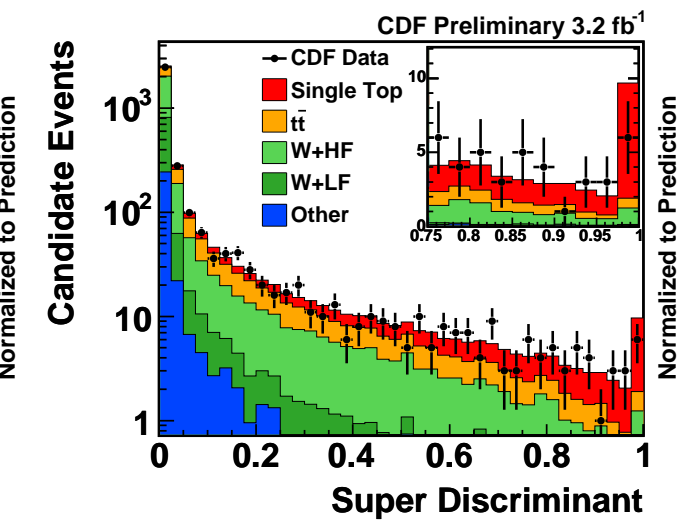

(b)

Figure 1: (a) Expected number of $\ell+E_{T}+$ jets events as a function of the number of jets for the signal (red) and each background process; the dashed band is the uncertainty in the background predictions. (b) CDF discriminant output distribution of the combination of the $\ell+E_{T}+$ jets analyses.

\section{References}

[1] B. W. Harris et al., Phys. Rev. D 66, 054024 (2002).

[2] Z. Sullivan, Phys. Rev. D 70, 114012 (2004);

J. Campbell, K. Ellis, and F. Tramontano, ibid. 70, 094012 (2004);

N. Kidonakis, ibid. 74, 114012 (2006).

[3] T. Aaltonen et al. (CDF Collaboration), Phys. Rev. Lett. 103, 092002 (2009).

[4] V. M. Abazov et al. (D0 Collaboration), Phys. Rev. Lett. 103, 092001 (2009).

[5] J. Lueck, Ph.D. thesis, University of Karlsruhe, FERMILAB-THESIS-2009-33 (2009).

[6] M. Feindt and U. Kerzel, Nucl. Instrum. Meth. A 559, 190 (2006).

[7] S. Richter, Ph.D. thesis, University of Karlsruhe, FERMILAB-THESIS-2007-35 (2007).

[8] P. Dong, Ph.D. thesis, University of California, Los Angeles, FERMILAB-THESIS-2008-12 (2008).

[9] S. Budd, Ph.D. thesis, Illinois University, Urbana, FERMILAB-THESIS-2008-30 (2008).

[10] K. Nakamura, Ph.D. thesis, Tsukuba University, FERMILAB-THESIS-2009-13 (2009).

[11] K. O. Stanley and R. Miikkulainen, Evolutionary Computation 10 (2) 99-127 (2002);

S. Whiteson and D. Whiteson, arXiv:hep-ex/0607012 (2006).

[12] C. Amsler et al. (Particle Data Group), Phys. Lett. B 667, 1 (2008).

[13] CDF public web page: http://www-cdf.fnal.gov/physics/new/top/public_singletop.html. 